\title{
Universality in Driven and Equilibrium Hard Sphere Liquid Dynamics
}

\author{
Lucas L. Treffenstädt and Matthias Schmidt \\ Theoretische Physik II, Physikalisches Institut, Universität Bayreuth, D-95447 Bayreuth, Germany
}

(Dated: 10 October 2020, to appear in Phys. Rev. Lett.)

\begin{abstract}
We demonstrate that the time evolution of the van Hove dynamical pair correlation function is governed by adiabatic forces that arise from the free energy and by superadiabatic forces that are induced by the flow of the van Hove function. The superadiabatic forces consist of drag, viscous, and structural contributions, as occur in active Brownian particles, in liquids under shear and in lane forming mixtures. For hard sphere liquids we present a power functional theory that predicts these universal force fields in quantitative agreement with our Brownian dynamics simulation results.
\end{abstract}

The van Hove function is arguably one of the most fundamental correlators that characterize the dynamical pair structure of a liquid on the microscopic scale $[1,2]$. It measures the probability of finding two particles at a distance $r$, where the particles are randomly chosen, but with a time lapse of duration $t$ between the two position measurements. Both the motion of the same particle, as well as spatio-temporal correlations of two distinct particles are captured. Significant physical insights into the dynamics of both simple and complex systems could be gained from studying their van Hove function. Examples thereof including cage formation in nematic and smectic liquid crystals [3], de Gennes narrowing of liquid iron [4], self-motion of water [5], the dynamics of colloidal hard spheres [6-8] and of colloid-polymer mixtures [9]. Experimentally, highly accurate results for the van Hove function are accessible based on microscopy of colloidal systems [6], as well as by scattering methods, which yield the Fourier transform $[2,5]$.

Much of our knowledge and understanding of the properties of the van Hove function is based on computer simulation work. Formulating a theoretical description for the complex spatial and temporal two-body dynamics remains a formidable challenge. Much insightful work has been carried out by Medina-Noyola and his coworkers on the basis of generalized Langevin equations [10-19], but also mode-coupling theory was used at high densities [20]. Furthermore, the closely related problem of identifying and studying memory kernels has received much recent attention in the context of of molecular dynamics [21-25].

The dynamical test particle limit [26-28] constitutes a formally exact reformulation of the time evolution of the van Hove function in a one-body picture. Instead of working explicitly with two-body correlations, an equivalent dynamical situation is constructed, where one-body profiles evolve in time, which offers significant conceptual simplification. Fixing a particle at the initial time at the origin establishes the equivalence with the original problem. The concept is formally exact, but it requires a prescription for the one-body dynamics to be useful in practice.

When choosing the dynamical density functional theory (DDFT) [29-31] to perform the one-body dynamics of the van Hove function $[6-8,26,27]$ one finds too rapid temporal decay of the interparticle correlations [32], as compared to benchmark data from Brownian dynamics (BD) computer simulations. This trend persists even when choosing Rosenfeld's fundamental measure theory [33-36] as an excellent approximation for the (hard sphere) free energy functional. Accounting for the observed reduction of particle mobility at increased density requires empirical adjustments to the DDFT framework $[6-8,27]$.

Power functional theory (PFT) [37] provides formally exact test particle dynamics [28], albeit very little explicit knowledge of the crucial superadiabatic force contributions [37, 38], i.e. those beyond DDFT, had originally been available [28]. In BD simulation work it was shown that the superadiabatic forces that govern the van Hove function are both significant in magnitude and nontrivial in their spatial and temporal structure [39]. In a variety of nonequilibrium systems, different superadiabatic force types were identified as providing the key mechanisms for prominent physical effects, such as the emergence of viscous and structural forces in BD flow [40-42], motilityinduced phase separation in active Brownian particles $[43,44]$, spontaneous lane formation in counter-driven mixtures [45], and memory-induced motion reversal [46].

Here we show that the identical types of superadiabatic forces that rule the behaviour of these driven systems, determine both qualitatively and quantitatively the van Hove function, and hence the intrinsic equilibrium dynamics. That the same form of superadiabatic forces apply across such a wide range of different physical situations indicates that the microscopic liquid dynamics are governed by universal mechanisms. Besides the conceptual importance of this finding, it allows concrete cross fertilization between results obtained for apparently very different systems.

Within the dynamical test particle limit, the van Hove function is expressed as a time-dependent one-body density profile $\rho(\mathbf{r}, t)$; here $\mathbf{r}$ indicates position and $t$ time. Often one splits into self and distinct parts, $\rho(\mathbf{r}, t)=$ $\rho_{\text {self }}(\mathbf{r}, t)+\rho_{\text {dist }}(\mathbf{r}, t)$. At the initial time the test ("self") particle is taken to be at the origin and the distinct particles are distributed according to the (static) pair correlation function $g(r)$ of the bulk liquid (as prescribed by Percus' static test particle limit [47]). Hence the initial conditions, at $t=0$, are $\rho_{\text {self }}(\mathbf{r}, 0)=\delta(\mathbf{r})$ and $\rho_{\text {dist }}(\mathbf{r}, 0)=\rho_{b} g(r)$, where $\delta(\cdot)$ indicates the Dirac delta function, $\rho_{b}$ is the bulk fluid number density, and $r=|\mathbf{r}|$. Figs. 1(a) and (b) depict an illustration. The dynam- 

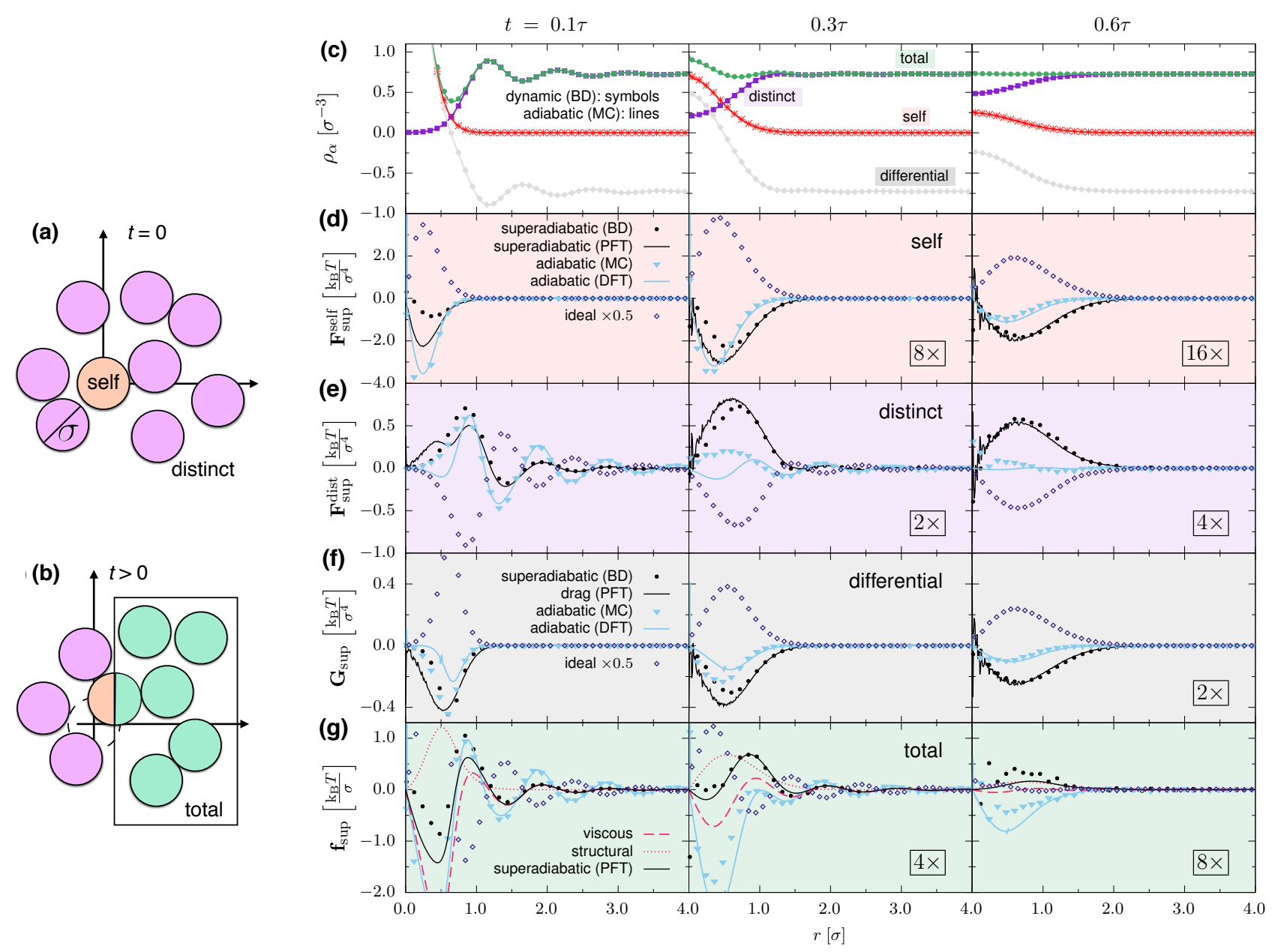

FIG. 1. (a) Illustration of the van Hove dynamical two-body correlation function in a bulk liquid of hard spheres of diameter $\sigma$ at time $t=0$ and (b) and $t>0$. (c)-(g) Results for the dynamical decay of the two-body structure of a bulk liquid of hard spheres at packing fraction 0.35 at times $t=0.1 \tau$ (left column), $0.3 \tau$ (middle column), and $0.6 \tau$ (right column) and as a function of the scaled distance $r / \sigma$. (c) Total $(\rho)$, self $\left(\rho_{\text {self }}\right)$, distinct $\left(\rho_{\text {dist }}\right)$, and differential $\left(\rho_{\Delta}\right)$ parts of the van Hove function, as indicated. The results from BD simulation (symbols) of the time evolution and from MC simulation (lines) of the corresponding adiabatic state coincide on the scale of the plot. (d) Self part of the superadiabatic force density $\mathbf{F}_{\text {sup }}^{\text {self }}$, as obtained from BD (symbols) and from PFT (solid line); also shown is the adiabatic self force density $\mathbf{F}_{\mathrm{ad}}^{\text {self }}$ from MC (blue symbols) and DFT (blue line). The ideal self force density, $-k_{B} T \nabla \rho_{\text {self }}$, is shown as a reference. (e) Distinct part of the superadiabatic force density, $\mathbf{F}_{\text {sup }}^{\text {dist }}$, as obtained from BD and from PFT. (f) Differential superadiabatic (drag) force density $\mathbf{G}_{\text {sup }}$, as obtained from BD (symbols) and from PFT (black line), and differential adiabatic force density $\mathbf{G}_{\mathrm{ad}}$, as arising from the adiabatic self correction. (g) Species-independent superadiabatic force field $\mathbf{f}_{\text {sup }}$ as obtained from BD and from PFT, along with the theoretical viscous $\left(\mathbf{f}_{\text {visc }}=-\rho^{-1} \delta P_{t}^{\text {visc }} / \delta \mathbf{v}\right)$ and structual contributions $\left(\mathbf{f}_{\text {struc }}=-\rho^{-1} \delta P_{t}^{\text {struc }} / \delta \mathbf{v}\right)$, where $\mathbf{f}_{\text {sup }}=\mathbf{f}_{\text {visc }}+\mathbf{f}_{\text {struc }}$. For the sake of clarity, the results in (d)-(g) at $t=0.3 \tau$ and $0.6 \tau$ are multiplied by a factor of $2 \times, 4 \times, 8 \times$ or $16 \times$, as indicated.

ics of the van Hove function are associated with timedependent one-body self and distinct currents, $\mathbf{J}_{\text {self }}(\mathbf{r}, t)$ and $\mathbf{J}_{\text {dist }}(\mathbf{r}, t)$, respectively. The total van Hove current is the sum $\mathbf{J}=\mathbf{J}_{\text {self }}+\mathbf{J}_{\text {dist }}$. A continuity equation holds for each species: $\partial \rho_{\alpha} / \partial t=-\nabla \cdot \mathbf{J}_{\alpha}$, where $\alpha=$ self, dist labels the two different species and $\nabla$ indicates the derivative with respect to $\mathbf{r}$. The partial one-body currents $\mathbf{J}_{\alpha}(\mathbf{r}, t)$ arise both from free diffusion and from internal interactions. Hence the one-body force density balance relation is

$$
\gamma \mathbf{J}_{\alpha}=-k_{B} T \nabla \rho_{\alpha}+\mathbf{F}_{\text {int }}^{\alpha},
$$

where $\gamma$ is the friction constant against the static background, $k_{B}$ is the Boltzmann constant, $T$ indicates absolute temperature, and $\mathbf{F}_{\text {int }}^{\alpha}(\mathbf{r}, t)$ is the internal force density distribution that acts on species $\alpha$. No external forces act in the bulk system, and the time-dependent (nonequilibrium) situation is solely introduced by the initial conditions $\rho_{\alpha}(\mathbf{r}, 0)$.

The internal force density consists of adiabatic $\left(\mathbf{F}_{\text {ad }}^{\alpha}\right)$ and superadiabatic ( $\left.\mathbf{F}_{\text {sup }}^{\alpha}\right)$ contributions [37, 38], according to the sum $\mathbf{F}_{\text {int }}^{\alpha}=\mathbf{F}_{\text {ad }}^{\alpha}+\mathbf{F}_{\text {sup }}^{\alpha}$. Here $\mathbf{F}_{\text {ad }}^{\alpha}(\mathbf{r}, t)$ is the force density distribution in an equilibrium ("adiabatic") system that is defined to possess one-body density pro- 
files $\rho_{\text {ad }}^{\alpha}(\mathbf{r})$ that are identical to those in the dynamical system at time $t: \rho_{\text {ad }}^{\alpha}(\mathbf{r}) \equiv \rho_{\alpha}(\mathbf{r}, t)$. This adiabatic construction is performed at each point in time, and hence $\rho_{\text {ad }}^{\alpha}(\mathbf{r})$ depends parametrically on $t$. The interparticle interaction potential in the adiabatic system is identical to that in the original dynamical system. The density distributions $\rho_{\mathrm{ad}}^{\alpha}(\mathbf{r})$ in the adiabatic system are stabilized by species-dependent external potentials $V_{\mathrm{ad}}^{\alpha}(\mathbf{r})$, which are guaranteed to exist in the adiabatic system due to the Mermin-Evans map of classical density functional theory (DFT) $[2,29]$.

The adiabatic force density can either be obtained by direct sampling in the adiabatic system [48], or, as we do here, from the force balance in the adiabatic system:

$$
\mathbf{F}_{\mathrm{ad}}^{\alpha}=k_{B} T \nabla \rho_{\alpha}+\rho_{\alpha} \nabla V_{\mathrm{ad}}^{\alpha},
$$

where all quantities on the right hand side are known. In practice we use a variant of the custom flow iterative method [48], where we sample the density profile at each iteration step using Monte Carlo, and adjust the external potentials $V_{\mathrm{ad}}^{\alpha}(\mathbf{r})$ accordingly [38] until the sampled density profiles in the adiabatic system match the dynamical ("target") density profiles $\rho_{\alpha}(\mathbf{r}, t)$.

Within classical density functional theory the adiabatic internal force density acting on species $\alpha$ is given by $\mathbf{F}_{\text {ad }}^{\alpha}(\mathbf{r}, t)=-\rho_{\alpha}(\mathbf{r}, t) \nabla \delta F_{\text {exc }} / \delta \rho_{\alpha}(\mathbf{r}, t)$, where $F_{\text {exc }}$ is the excess (over ideal gas) intrinsic Helmholtz free energy functional. For the case of hard spheres, Rosenfeld's fundamental measure theory [33-36] constitutes an excellent approximation for $F_{\text {exc }}$. We furthermore use the "quenched" approach by Stopper et al. [8], where a self-correction is applied in order to account for the fact that the self density profile represents a single particle sharply (rather than a grand ensemble average). This approach avoids having to use canonical decomposition $[49,50]$ in order to generate results that are specific to fixed particle number.

Within PFT the superadiabatic force density is obtained from a functional derivative of the superadiabatic excess free power functional $P_{t}^{\text {exc }}$ according to

$$
\mathbf{F}_{\text {sup }}^{\alpha}(\mathbf{r}, t)=-\frac{\delta P_{t}^{\operatorname{exc}}}{\delta \mathbf{v}_{\alpha}(\mathbf{r}, t)},
$$

where the derivative is taken at fixed density profiles, and the species-resolved one-body velocity profile is $\mathbf{v}_{\alpha}(\mathbf{r}, t)=\mathbf{J}_{\alpha}(\mathbf{r}, t) / \rho_{\alpha}(\mathbf{r}, t)$. As an approximation we use a functional that consists of drag [43-45], viscous [40-42, 45, 46], and structural [41, 42, 44] contributions, $P_{t}^{\text {exc }}=P_{t}^{\text {drag }}+P_{t}^{\text {visc }}+P_{t}^{\text {struc }}$, according to

$$
\begin{aligned}
P_{t}^{\text {exc }}=\frac{C_{\text {drag }}}{2} \int d \mathbf{r} \rho_{\text {self }} \rho_{\text {dist }}\left(\mathbf{v}_{\text {self }}-\mathbf{v}_{\text {dist }}\right)^{2} \\
+\int d \mathbf{r} d \mathbf{r}^{\prime} \int_{0}^{t} d t^{\prime} n_{3} n_{3}^{\prime}(\nabla \cdot \mathbf{v})\left(\nabla^{\prime} \cdot \mathbf{v}^{\prime}\right) K_{\text {visc }} \\
\quad-\int d \mathbf{r} d \mathbf{r}^{\prime} \int_{0}^{t} d t^{\prime}\left(n_{3}^{\prime} \mathbf{v}^{\prime}\right)^{2}(\nabla \cdot \mathbf{J}) K_{\text {struc }}
\end{aligned}
$$

where $C_{\mathrm{drag}}$ is a constant and the kernels $K_{e}(\Delta \mathbf{r}, \Delta t)$, where $e=$ visc, struc, depend on the relative spatial and temporal distances $\Delta \mathbf{r}=\mathbf{r}-\mathbf{r}^{\prime}$ and $\Delta t=t-t^{\prime}$; the local packing fraction $n_{3}(\mathbf{r}, t)$ is obtained by convolution with $\rho(\mathbf{r}, t)[33-36] ; \nabla^{\prime}$ indicates the derivative with respect to $\mathbf{r}^{\prime}$, and we use the shorthand $n_{3}^{\prime} \equiv n_{3}\left(\mathbf{r}^{\prime}, t^{\prime}\right)$ and $\mathbf{v}^{\prime} \equiv$ $\mathbf{v}\left(\mathbf{r}^{\prime}, t^{\prime}\right)$. Here the total microscopic velocity profile is $\mathbf{v}=\mathbf{J} / \rho$. We use the diffusing memory form [46] for $K_{e}(\Delta \mathbf{r}, \Delta t)$, which consists of a product of a constant $C_{e}$ that controls the overall strength, an exponential decay with decay time constant $\tau_{e}$ and a diffusing Gaussian with diffusion constant $D_{e}$. Explicitly the form is

$$
K_{e}(\Delta \mathbf{r}, \Delta t)=\frac{C_{e} \exp \left(-\Delta \mathbf{r}^{2} /\left(4 D_{e} \Delta t\right)-\Delta t / \tau_{e}\right)}{\left(4 \pi D_{e} \Delta t\right)^{3 / 2} \tau_{e}} .
$$

The derivative (3) when applied to (4), yields an explicit expression for $\mathbf{F}_{\text {sup }}^{\alpha}$, which we evaluate below, using BD data for $\rho_{\alpha}$ and $\mathbf{v}_{\alpha}$ as input. We choose the following set of parameters: The drag strength is $C_{\mathrm{drag}}=2.2 \gamma \sigma^{3}$. The values for viscous memory kernel are identical to those used in Ref. [46]: $C_{\text {visc }}=5.8 k_{B} T /\left(\sigma^{3} \tau\right), D_{\text {visc }}=$ $5.6 \sigma^{2} / \tau$, and $\tau_{\text {visc }}=0.02 \tau$. The structural memory kernel has $C_{\text {struc }}=0.42 k_{B} T \tau^{2} / \sigma^{2}, D_{\text {struc }}=0.25 \sigma^{2} / \tau$, and $\tau_{\text {struc }}=0.8 \tau$. Here the natural (Brownian) time scale is $\tau=\gamma \sigma^{2} /\left(k_{B} T\right)$, where $\sigma$ is the hard sphere diamater. For standard colloids with diameter $\sigma=1 \mu \mathrm{m}$ dispersed in water at $T=20^{\circ} \mathrm{C}$, using the Stokes-Einstein form for $\gamma=3 \pi \eta \sigma$ [2] gives $\gamma=9.44 \cdot 10^{-13} \mathrm{~kg} \mathrm{~s}^{-1}$, which yields $\tau=2.33 \mathrm{~s}$. The corresponding memory times are $\tau_{\text {visc }}=0.046 \mathrm{~s}$ and $\tau_{\text {struc }}=1.87 \mathrm{~s}$, i.e. values that are well inside of an experimentally accessible range. Using larger colloids $[6,51]$ scales up the values for the memory times accordingly; the particles used e.g. for the (twodimensional) system of Ref. [6] are of size $\sigma=4.04 \mu \mathrm{m}$, which correspondingly upscales the values for both memory times by a factor of four.

In order to gain further insight into the nature of the relevant forces, we follow Ref. [45] and rewrite the internal forces that act in a binary mixture as consisting of a non-selective ("differential") force field $\left(\mathbf{f}_{\text {int }}\right)$ and a selective ("total") force density $\left(\mathbf{G}_{\text {int }}\right)$, such that the self and distinct internal forces density distributions can respectively be expressed as

$$
\mathbf{F}_{\text {int }}^{\text {self }}=\rho_{\text {self }} \mathbf{f}_{\text {int }}+\mathbf{G}_{\text {int }}, \quad \mathbf{F}_{\text {int }}^{\text {dist }}=\rho_{\text {dist }} \mathbf{f}_{\text {int }}-\mathbf{G}_{\text {int }} .
$$

Using the new fields $\mathbf{f}_{\text {int }}$ and $\mathbf{G}_{\text {int }}$ in the partial force density balance (1) leads to equations of motion for the total and for the "differential" motion,

$$
\begin{aligned}
\gamma \mathbf{v} & =-k_{B} T \nabla \ln \rho+\mathbf{f}_{\text {int }}, \\
\gamma \mathbf{J}_{\Delta} & =-k_{B} T \nabla \rho_{\Delta}+\rho_{\Delta} \mathbf{f}_{\text {int }}+2 \mathbf{G}_{\text {int }},
\end{aligned}
$$

where the differential van Hove current is $\mathbf{J}_{\Delta}=\mathbf{J}_{\text {self }}-$ $\mathbf{J}_{\text {dist }}$, and the differential van Hove function is $\rho_{\Delta}=$ $\rho_{\text {self }}-\rho_{\text {dist }}$. Solving the linear set of equations (6) yields

$$
\mathbf{f}_{\text {int }}=\mathbf{F}_{\text {int }} / \rho, \quad \mathbf{G}_{\text {int }}=\left(\rho_{\text {dist }} \mathbf{F}_{\text {int }}^{\text {self }}-\rho_{\text {self }} \mathbf{F}_{\text {int }}^{\text {dist }}\right) / \rho,
$$


which allows to obtain results for $\mathbf{f}_{\text {int }}(\mathbf{r}, t)$ and $\mathbf{G}_{\text {int }}(\mathbf{r}, t)$ [from the correlators on the right hand sides of (9). Due to the linearity of the transformations (9), splitting into adiabatic and superadiabatic contributions applies according to $\mathbf{f}_{\text {int }}=\mathbf{f}_{\text {ad }}+\mathbf{f}_{\text {sup }}$ and $\mathbf{G}_{\text {int }}=\mathbf{G}_{\text {ad }}+\mathbf{G}_{\text {sup }}$.

Figure 1(c) presents results for the van Hove function of hard spheres. Shown are the self and the distinct part, $\rho_{\text {self }}(r, t)$ and $\rho_{\text {dist }}(r, t)$, at three different representative times $t / \tau=0.1,0.3,0.6$. The results are obtained using event-driven Brownian dynamics (BD) computer simulations [52]. We use $N=1090$ particles in a threedimensional simulation box of size $15 \times 10 \times 10 \sigma^{3}$. The sampling is based on $10^{6}$ time steps of size $10^{-3} \tau$, and hence an overall simulation time of $10^{3} \tau$. Appropriate filling of histograms of particle pair distances yields results for the van Hove function.

At the early time, $t=0.1 \tau$ (first column of Fig. 1), the van Hove function has moderately decayed, as compared to its initial condition. Over the course of time, cf. the results for $0.3 \tau$ (middle column) and $0.6 \tau$ (right column), the self part broadens and its height correspondingly decreases. The initial correlation hole in the distinct van Hove function is gradually being filled. Besides these transport processes, the initially pronounced oscillations at distances $r \gtrsim \sigma$ decay.

We demonstrate the agreement of adiabatic and dynamical density profiles in Fig. 1(c), by showing the MC simulation results obtained from equilibrium sampling of the adiabatic state, i.e. of the system in which the external potential $V_{\text {ad }}^{\text {self }}(\mathbf{r})$ acts on the (single) self particle and $V_{\text {ad }}^{\text {dist }}(\mathbf{r})$ acts on the remaining $N-1$ particles. (As $N$ is large enough, we do not expect that finite size effects are relevant.) Apart from very small numerical artifacts, clearly the agreement of dynamical and adiabatic density profiles is excellent. Hence we trust results for the adiabatic force densities (presented below), obtained via (2).

Besides the self and distinct parts, we also show results for the total van Hove function, $\rho=\rho_{\text {self }}+\rho_{\text {dist }}$, and the differential van Hove function $\rho_{\Delta}=\rho_{\text {self }}-\rho_{\text {dist }}$ in Fig. 1(c). Clearly the spatial structuring of $\rho$ is much reduced upon disregarding the self-distinct labelling. Nevertheless, as all particles in the system are ultimately identical, and the self-distinct labelling was introduced for mere book-keeping purposes, one might wonder whether the physically most relevant phenomena are revealed or are rather hidden by the labelling.

In Fig. 1(d) we show results for the different contributions to the self force density. As a reference, we plot the ideal contribution, $-k_{B} T \nabla \rho_{\text {self }}$, which tends to spread the self peak in time. Here positive (negative) values both of force fields and of force densities indicate the outward (inward) direction. The adiabatic force density counteracts the ideal part, and hence tends to stabilize the self density peak. The DFT results yield very satisfactory results, as compared to the MC data, at all times considered. The superadiabatic self force density supports the effect of $\mathbf{F}_{\text {ad }}^{\text {self }}$, but it has longer range and larger magnitude at later times. Except for a slight overestimation at $0.1 \tau$, the PFT reproduces this effect very well, and hence provides a mechanism for the slowing down of the dynamics.

The contributions to the distinct force density, shown in Fig. 1(e), show more complex, oscillatory behaviour, at both earlier times. The ideal force density is again directly related, via the spatial derivative, to the distinct density, shown in Fig. 1(c). The oscillations of the distinct density profiles are hence imprinted into ideal force density and their effect is to homogenize the density. As is the case for the self part, the adiabatic force density counteracts this effect, and hence tends to stabilize the density oscillations. The DFT results for $\mathbf{F}_{\text {ad }}^{\text {self }}$ are very satisfactory, with some underestimation inside of the core, $r \lesssim \sigma$. The superadiabatic distinct force density has complex spatial features. It tends to slow down the decay of the spatial structure. At early times, the magnitude is smaller than that of $\mathbf{F}_{\mathrm{ad}}^{\text {dist }}$, but this relationship changes at later times, where $\mathbf{F}_{\text {sup }}^{\text {dist }}$ becomes dominant. Again, up to some deviations inside of the core, the PFT describes $\mathbf{F}_{\text {sup }}^{\text {dist }}$ in very good agreement with the BD data.

In Fig. 1(f) we show results for the contributions to the differential force density, $\mathbf{G}_{\mathbf{i n t}}$, as defined in (9) and being relevant for the differential equation of motion (8). Within the PFT, we can clearly identify that $\mathbf{G}_{\text {sup }}$ is solely due to the drag effect, i.e. the friction generated by the interflow of the self and distinct components. This result is relevant for the hard sphere dynamics at long times. Taking only the drag force as the dominant internal effect and balancing it with ideal diffusion, the long time self diffusion coefficient follows as $D_{L}=k_{B} T /\left(\gamma+\rho_{b} C_{\mathrm{drag}}\right)$. Within this approximation we obtain $D_{L}=0.38 \sigma^{2} / \tau\left(=0.16 \mu \mathrm{m}^{2} / \mathrm{s}\right.$ for $\sigma=1 \mu \mathrm{m}$ and $\tau=2.33 \mathrm{~s}$ as above), in very reasonable agreement with our bare simulation result of $D_{L}=0.32 \sigma^{2} / \tau(=$ $\left.0.14 \mu \mathrm{m}^{2} / \mathrm{s}\right)$. In contrast, the motion of the total van Hove function, for which we show the relevant force fields in Fig. 1(g), is due to both compressional viscosity and structural forces, with both complex spatial and temporal behaviour, which are well captured by the PFT. Crucially, while the details of the superadiabatic force fields vary depending on the type of dynamical situation considered, regarding these as arising from a kinematic functional (4) reveals their universal characteristics.

In conclusion, we have traced the mechanisms that govern the time evolution of the van Hove function for hard spheres by identifying three different and universal types of nonequilibrium force contributions, all of which have been shown previously to be relevant across a broad spectrum of nonequilibrium and driven systems. The forces are due to i) drag of the tagged ("self") particle against the surrounding fluid of distinct particles, ii) volume (or "bulk") viscosity due to the correlation shells undergoing compressional-expansional flow, and iii) structural nonequilibrium effects, which stabilize the spatial liquid structure against decay. The power functional approximation generates all three types of nonequilibrium force fields in quantitative agreement with Brownian dy- 
namics computer simulation results. Our results hence demonstrate intimate interrelationships between equilibrium and nonequilibrium hard sphere properties.

It would be interesting to investigate in future work the relationship of our findings to Rosenfeld's excess entropy scaling $[53,54]$, as much advanced by Truskett and his coworkers $[55,56]$, to the nonequilibrium OrnsteinZernike framework [57, 58], as well as to the findings by Dyre and coworkers on universality across systems with different interparticle interaction potentials $[59,60]$.

\section{ACKNOWLEDGMENTS}

We thank Daniel de las Heras and Sophie Hermann for useful comments. This work is supported by the German Research Foundation (DFG) via project number 317849184 .
[1] Correlations in space and time and Born approximation scattering in systems of interacting particles. L. van Hove, Phys. Rev. 95, 249 (1954).

[2] J. P. Hansen and I. R. McDonald, Theory of Simple Liquids, 4th ed. (Academic Press, London, 2013).

[3] Self-Diffusion of Particles in Complex Fluids: Temporary Cages and Permanent Barriers. M. Bier, R. van Roij, M. Dijkstra, and P. van der Schoot, Phys. Rev. Lett. 101, 215901 (2008).

[4] Atomic dynamics in simple liquid: de Gennes narrowing revisited. B. Wu, T. Iwashita, and T. Egami, Phys. Rev. Lett. 120, 135502 (2018).

[5] Local self-motion of water through the van Hove function. Y. Shinohara, W. Dmowski, T. Iwashita, D. Ishikawa, A. Q. R. Baron, and T. Egami, Phys. Rev. E 102, 032604 (2020).

[6] Bulk dynamics of Brownian hard disks: Dynamical density functional theory versus experiments on twodimensional colloidal hard spheres. D. Stopper, A. Thorneywork, R. P. A. Dullens, and R. Roth, J. Chem. Phys. 148, 104501 (2018).

[7] Modeling diffusion in colloidal suspensions by dynamical density functional theory using fundamental measure theory of hard spheres. D. Stopper, K. Marolt, R. Roth, and H. Hansen-Goos, Phys. Rev. E 92, 022151 (2015).

[8] Communication: Dynamical density functional theory for dense suspensions of colloidal hard spheres. D. Stopper, R. Roth, and H. Hansen-Goos, J. Chem. Phys. 143, 181105 (2015).

[9] Structural relaxation and diffusion in a model colloidpolymer mixture: dynamical density functional theory and simulation. D. Stopper, R. Roth, and H. HansenGoos, J. Phys.: Condens. Matter 28, 455101 (2016).

[10] Overdamped van Hove function of colloidal suspensions. L. Yeomans-Reyna and M. Medina-Noyola, Phys. Rev. E 62, 3382 (2000).

[11] Van Hove function of colloidal mixtures: Exact results. M. A. Chávez-Rojo and M. Medina-Noyolaa, Physica A 366, 55 (2006).

[12] The overdamped van Hove function of atomic liquids. L. López-Flores, L. L. Yeomans-Reyna, Martin ChávezPáez, and M. Medina-Noyola, J. Phys.: Condens. Matter 24375107 (2012).

[13] Dynamic equivalences in the hard-sphere dynamic universality class. L. López-Flores, H. Ruiz-Estrada, M. Chávez-Páez, and M. Medina-Noyola, Phys. Rev. E 88, 042301 (2013).

[14] Self-consistent generalized Langevin equation theory of the dynamics of multicomponent atomic liquids. E. Lázaro-Lázaro, P. Mendoza-Méndez, L. F. ElizondoAguilera, J. A. Perera-Burgos, P. E. Ramirez-González, G. Pérez-Angel, R. Castaneda-Priego, and M. MedinaNoyola, J. Chem. Phys. 146, 184506 (2017).

[15] Self-consistent theory of collective Brownian dynamics: Theory versus simulation. L. Yeomans-Reyna, H. AcunaCampa, F. de Jesus Guevara-Rodriguez, and M. MedinaNoyola, Phys. Rev. E 67, 021108 (2003).

[16] Dynamic arrest within the self-consistent generalized Langevin equation of colloid dynamics. L. YeomansReyna, M. A. Chávez-Rojo, P. E. Ramirez-González, R. Juárez-Maldonado, M. Chávez-Páez, and M. MedinaNoyola, Phys. Rev. E 76, 041504 (2007).

[17] Simplified self-consistent theory of colloid dynamics. R. Juárez-Maldonado, M. A. Chávez-Rojo, P. E. RamirezGonzález, L. Yeomans-Reyna, and M. Medina-Noyola, Phys. Rev. E 76, 062502 (2007).

[18] Non-equilibrium relaxation and near-arrest dynamics in colloidal suspensions. M. Medina-Noyola, and P. Ramirez-González, J. Phys.: Condens. Matter 21, 504103 (2009).

[19] Aging of a homogeneously quenched colloidal glassforming liquid. P. Ramirez-González and M. MedinaNoyola, Phys. Rev. E 82, 061504 (2010).

[20] Structural relaxation of polydisperse hard spheres: Comparison of the mode-coupling theory to a Langevin dynamics simulation. F. Weysser, A. M. Puertas, M. Fuchs, and Th. Voigtmann, Phys. Rev. E 82, 011504 (2010).

[21] Molecular hydrodynamics from memory kernels. D. Lesnicki, R. Vuilleumier, A. Carof, and B. Rotenberg, Phys. Rev. Lett. 116, 147804 (2016).

[22] Microscopic flow around a diffusing particle. D. Lesnicki and R. Vuilleumier, J. Chem. Phys. 147, 094502 (2017).

[23] Computing bulk and shear viscosities from simulations of fluids with dissipative and stochastic interactions. G. Jung and F. Schmid, J. Chem. Phys. 144, 204104 (2016).

[24] Iterative reconstruction of memory kernels. G. Jung, M. Hanke, and F. Schmid, J. Chem. Theo. Comput. 13, 2481 (2017).

[25] Generalized Langevin dynamics: construction and numerical integration of non-Markovian particle-based models. G. Jung, M. Hanke, and F. Schmid, Soft Matter 14, 9368 (2018).

[26] Dynamics in inhomogeneous liquids and glasses via the test particle limit. A. J. Archer, P. Hopkins, and M. Schmidt, Phys. Rev. E 75, 040501(R) (2007).

[27] The van Hove distribution function for Brownian hard 
spheres: Dynamical test particle theory and computer simulations for bulk dynamics. P. Hopkins, A. Fortini, A.J. Archer, and M. Schmidt, J. Chem. Phys. 133, 224505 (2010).

[28] Power functional theory for the dynamic test particle limit. J. M. Brader and M. Schmidt, J. Phys.: Condens. Matter 27, 194106 (2015).

[29] The nature of the liquid-vapour interface and other topics in the statistical mechanics of non-uniform, classical fluids. R. Evans, Adv. Phys. 28, 143 (1979).

[30] Dynamic density functional theory of fluids. U. M. B. Marconi and P. Tarazona, J. Chem. Phys. 110, 8032 (1999).

[31] Dynamical density functional theory and its application to spinodal decomposition. A. J. Archer and R. Evans, J. Chem. Phys. 121, 4246 (2004).

[32] Dynamics of localized particles from density functional theory. J. Reinhardt and J. M. Brader, Phys. Rev. E 85, 011404 (2012).

[33] Free-energy model for the inhomogeneous hard-sphere fluid mixture and density-functional theory of freezing, Y. Rosenfeld, Phys. Rev. Lett. 63, 980 (1989).

[34] Density functional theories of hard particle systems. P. Tarazona, J.A. Cuesta, and Y. Martinez-Raton, Lect. Notes Phys. 753, 247 (2008).

[35] Fundamental measure theory for hard-sphere mixtures: a review. R. Roth, J. Phys.: Condens. Matt. 22, 063102 (2010).

[36] Recent developments in classical density functional theory. J. F. Lutsko, Adv. Chem. Phys. 144, 1 (2010).

[37] Power functional theory for Brownian dynamics. M. Schmidt and J. M. Brader, J. Chem. Phys. 138, 214101 (2013).

[38] Superadiabatic forces in Brownian many-body dynamics. A. Fortini, D. de las Heras, J. M. Brader, and M. Schmidt, Phys. Rev. Lett. 113, 167801 (2014).

[39] Dynamic pair correlations and superadiabatic forces in a dense Brownian liquid. T. Schindler and M. Schmidt, J. Chem. Phys. 145, 064506 (2016).

[40] Velocity gradient power functional for Brownian dynamics. D. de las Heras and M. Schmidt, Phys. Rev. Lett. 120, 028001 (2018).

[41] Structural nonequilibrium forces in driven colloidal systems. N. C. X. Stuhlmüller, T. Eckert, D. de las Heras, and M. Schmidt, Phys. Rev. Lett. 121, 098002 (2018).

[42] Flow and structure in nonequilibrium Brownian manybody systems. D. de las Heras and M. Schmidt, Phys. Rev. Lett. 125, 018001 (2020).

[43] Nonequilibrium phase behaviour from minimization of free power dissipation. P. Krinninger, M. Schmidt, and J. M. Brader, Phys. Rev. Lett. 117, 208003 (2016).

[44] Non-negative interfacial tension in phase-separated ac- tive Brownian particles. S. Hermann, D. de las Heras, and M. Schmidt, Phys. Rev. Lett. 123, 268002 (2019).

[45] Superadiabatic demixing in nonequilibrium colloids. T. Geigenfeind, D. de las Heras, and M. Schmidt, Comms. Phys. 3, 23 (2020).

[46] Memory-induced motion reversal in Brownian liquids. L. L. Treffenstädt and M. Schmidt, Soft Matter 16, 1518 (2020).

[47] Approximation methods in classical statistical mechanics. J. K. Percus, Phys. Rev. Lett. 8, 462 (1962).

[48] Custom flow in overdamped Brownian Dynamics. D. de las Heras, J. Renner, and M. Schmidt, Phys. Rev. E 99, 023306 (2019).

[49] Full canonical information from grand potential density functional theory. D. de las Heras and M. Schmidt, Phys. Rev. Lett. 113, 238304 (2014).

[50] Particle conservation in dynamical density functional theory. D. de las Heras, J. M. Brader, A. Fortini, and M. Schmidt, J. Phys.: Condens. Matter 28, 244024 (2016).

[51] Non-equilibrium sedimentation of colloids on the particle scale. C. P. Royall, J. Dzubiella, M. Schmidt, and A. van Blaaderen, Phys. Rev. Lett. 98, 188304 (2007).

[52] Event-driven Brownian dynamics for hard spheres. A. Scala, T. Voigtmann, and C. De Michele, J. Chem. Phys. 126, 134109 (2007).

[53] Relation between the transport coefficients and the internal entropy of simple systems. Y. Rosenfeld, J. Chem. Phys. 149, 210901 (2018).

[54] Perspective: Excess-entropy scaling. J. C. Dyre, J. Chem. Phys. 149, 210901 (2018).

[55] Quantitative link between single-particle dynamics and static structure of supercooled liquids. J. Mittal, J. R. Errington, and T. M. Truskett, J. Phys. Chem. B Lett. 110, 18147 (2006).

[56] Communication: Generalizing Rosenfeld's excessentropy scaling to predict long-time diffusivity in dense fluids of Brownian particles: From hard to ultrasoft interactions. M. J. Pond, J. R. Errington, and T. M. Truskett, J. Chem. Phys. 134, 081101 (2011)

[57] Nonequilibrium Ornstein-Zernike relation for Brownian many-body dynamics. J. M. Brader and M. Schmidt, J. Chem. Phys. 139, 104108 (2013).

[58] Dynamic correlations in Brownian many-body systems. J. M. Brader and M. Schmidt, J. Chem. Phys. 140, 034104 (2014).

[59] Simple liquids' quasiuniversality and the hard-sphere paradigm. J. C. Dyre, J. Phys.: Condens. Matter 28, 323001 (2016).

[60] Revisiting the Stokes-Einstein relation without a hydrodynamic diameter. L. Costigliola, D. M. Heyes, T. B. Schrøder, and J. C. Dyre J. Chem. Phys. 150, 021101 (2019). 